\title{
STRUCTURAL MECHANICS: HOW COMPUTERS AFFECTED IT
}

\author{
LEONE CORRADI DELL'ACQUA (*)
}

\begin{abstract}
SunTo. - L'avvento del calcolo elettronico ha avuto un effetto profondo su molti aspetti della nostra vita e, ovviamente, anche la meccanica strutturale ne è rimasta influenzata. L'impatto è stato così significativo che alcuni hanno profetizzato una vera e propria rivoluzione, l'avvento di una meccanica "discreta" che sostituisse la tradizionale formulazione continua. Oggi, dopo alcuni anni, possiamo verificare che tale rivoluzione non è avvenuta. Ciò tuttavia non significa che la meccanica strutturale sia rimasta inalterata: il calcolo numerico ha suscitato una revisione critica che, se non ha alterato i fondamenti della disciplina, ha comunque introdotto alcune chiarificazioni e miglioramenti.
\end{abstract}

ABSTRACT. - The advent of electronic computation had a profound effect on many aspects of present life and, obviously, structural mechanics also was affected. The impact was so significant that some researchers predicted a sort of revolution, the advent of a "discrete" mechanics that would replace the traditional continuum formulation. A few years have elapsed since then and we can state that that such a revolution did not occur as yet. Nevertheless, structural mechanics did not remain unaltered: computations gave rise to a critical review of some aspects which certainly did not alter the foundations of the field, but contributed to clarify some points and improve the overall picture under some respect.

\section{INTRODUCTION}

The mathematical theory of elasticity, established at the beginning of the nineteenth century, permits the formulation of the elastic problem

(*) Istituto Lombardo Accademia di Scienze e Lettere, Politecnico Milano, Italia. E-mail: leonemaria.corradidellacqua@fastwebnet.it 
for a general solid, at least within the small strain framework. The equations it involves, however, are difficult to handle. A number of solutions of specific problems were produced by several researches but these solutions, even if important, are far from satisfying most engineering needs. A considerable amount of effort was also devoted to the search for reasonable approximations making the problem mathematically easier to solve. Conscious assumptions on the deformation of solids exhibiting a specific structure, such as beams, plates or shells, permitted significant simplifications, even if solution difficulties were not completely eliminated. In fact it was only for frames that a general formulation involving algebraic equations was produced. If linear, a system of algebraic equations can be easily solved in principle. However, when only hand computations or, at most, mechanical calculators were available, the solution of structures of significant size remained out of reach.

The situation has drastically changed when electronic computers entered the scene. As soon as computers became available to a large part of the scientific community, their use for structural computations was envisaged. A new field, known as Computational Mechanics arose and its success gave rise to great expectations and some concern. Some people predicted the advent of a completely new mechanics, of discrete nature, which would replace the traditional continuum mechanics, or at least represent an alternative to it. At present such a dream did not come true: early developments of computational mechanics were well rooted on sound classical mechanics bases. Further progress, however, gave rise to new ideas, which did not change structural mechanics but contributed to clarify and improve it under some respect. This contribution aims at discussing some of these aspects.

\section{A “MATTER OF MATHEMATICAL CALCULATION”}

In the introduction to his classical Treatise on the mathematical theory of elasticity, Love makes the following statement: "When the general equations bad been obtained, all questions of the small strain of elastic bodies were reduced to a matter of mathematical calculation" ([1], p. 2). That "matter of mathematical calculation" represented a serious obstacle before the advent of computers and much effort in structural mechanics research was devoted to attempts at overcoming it.

In fact, closed form solutions of elastic problems were obtained 
for a limited number of specific problems only. Such solutions, no doubt important, were unable to satisfy several situations of engineering interest ad much effort in research was devoted to the search for reasonable approximations. By translating from [2] one can state that "one of the greatest merits of nineteenth century engineers was their capability of deducing from the rigorous but unusable results of the great preceding scientists, simple and versatile theories, consciously based on drastic simplifying bypotheses, without betraying the physical nature of the structural bebavior".

One of the ideas that were introduced is that the average values of stresses can be considered as meaningful in some instances. On this idea are based the plane stress formulation and the approximate solutions given by Bredt and Jourawsky for the torsion and shear problems, respectively. Also the structural theories of beams, plates and shells, which provide the stress field in terms of resultants (axial and shear forces, bending and twisting moments) are based on the same concept. They assume as starting point kinematic hypotheses suggested by the particular structure of the problem (e.g., cross sections of a beam remain plane) and equilibrium is imposed on the average. The procedure was explicitly employed by Kirchhoff when formulating his theory of thin plates [3]. The starting assumption was that straight segments orthogonal to the middle surface keep straight and orthogonal to the middle surface during the deformation process and the strain field and the strain-displacement relations were derived on this basis. Then, equilibrium was enforced by exploiting the principle of virtual work. Some of the results (in particular, equilibrium boundary conditions) might appear cumbersome, but they are consistent with the displacement field and the resulting formulation is mathematically sound.

The results above often succeeded in replacing the original formulation with a simpler one and a number of solutions of practical interest were obtained on their basis, but for problems such as plates and shells solutions could be computed only for specific shapes and boundary conditions. Only for frames and trusses a general formulation in terms of algebraic equations was obtained. If linear, a system of simultaneous algebraic equations could be solved easily, but as long as only hand computations were possible or, at most, desk calculators were available, the solution of problems of significant size were impractical and the effort was limited to very unusual or very important situations. 


\section{The advent of Computational Mechanics}

The situation changed when computers became available to the scientific community and a new discipline, known as Computational Mechanics, arose. Conventionally, the finite element method for structural analysis is considered to have been initiated by the paper published by Turner, Clough, Martin and Topp in 1956 [4]. Such a precise date of birth could appear surprising. In fact, if the content of the paper were enucleated from the computational context of the time, its importance would be greatly underestimated. According to [5] the idea of a matrix approach to structural analysis was apparently suggested by Edward Study already in 1903, though it attracted little attention at the time because of the amount of computation involved. Discrete models able to represent, at least in principle, a variety of geometrical shapes and load conditions also were previously proposed (see, e.g. [6]). Moreover, the procedure used to establish the matrix relations governing the element behavior was based on ingenuity and engineering judgment rather than on mechanically sound concepts and hardly can be considered as a starting point for future developments.

However only two years earlier an event took place, marking a turning point in the use of computing machinery. At the time, computers had been available by some years, but programming had to be performed in a (often machine dependent) machine language. Mastering the code relevant to each computer required a long and tedious training, which had to be restarted from scratch with any change in the machinery and, as a consequence, programming was regarded as a professional job. It was only in 1954 that IBM released the first FORmula TRANslator language (FORTRAN) permitting relations with computers through an essentially standard mathematical symbolism. Symbolic languages transformed computers into practical tools effectively available to the entire scientific community and matrix approaches could be regarded as feasible solution methods, flexible enough to tackle a variety of structural typologies, geometrical shapes and load conditions. In this context, the aforementioned paper appeared as the first proposal on this line.

The development of computational mechanics was astoundingly rapid. After a short initial period in which more or less heuristic considerations, largely based on engineering experience and ingenuity, were employed, the formulation of the problem was brought back to 
sound mechanical bases. The first approach used is the so-called displacement formulation, which still is the best understood and most widely used. The main reason for its success is that it is based on assumptions that are not only simple, but also in a sense natural and easily accepted by engineers. In fact, as in the structural theories of beams plates and shells, the formulation is based on the assumption of a suitable displacement model. The only difference is that the assumption is not a-priori accepted because considered as reasonable; rather it is dictated by the requirement that the solution converges to the continuous one as the model is refined. Once the displacement model is defined, the same procedure employed by Kirchhoff for his plate formulation is used.

Even if it shares many of the features of structural theories, the finite element method has some special characteristics, aiming at the exploitation of the facilities offered by high speed computers. In one of the first books written on the subject, Przemieniecki makes the following statement: "Matrix methods are based on the concept of replacing the actual continuous structure by a mathematical model made up of from structural elements of finite size ... having known elastic and inertial properties that can be expressed in matrix form. The matrices representing these properties are considered as building blocks, which, when fitted together according to a set of rules derived from the theory of elasticity, provide the static and dynamic properties of the actual structural system" [7]. Central feature of the method is the idea of building blocks fitted together or, in a presently more familiar language, that of the assemblage of finite element matrices, an operation that computers perform efficiently on the basis of limited input information. The properties of finite elements, however, are calculated using the theory of continuous elastic media and continuum mechanics concepts are used to formulate the overall behavior of their assemblage.

Thus, the displacement finite element formulation is similar under many respects to engineering structural theories and it shares their effectiveness and simplicity. However, it shares also their drawbacks. One is the excess of stiffness: the classical theories of beams, plates and shells, in their simplest formulations that neglect transverse shear strains, overestimate the structural stiffness; in finite elements this effect sometimes is so strong that is referred to as locking. It occurs especially for incompressible bodies because the displacement model often has a limited capability in representing isochoric deformations. A 
second problem is related to the representation of local stresses: in structural theories the information on stresses is provided in terms of resultants (or generalized variables), which is adequate in most cases; in displacement finite element models generalized stresses do not possess any physical meaning and are useless, to the point that they are not even computed. Stresses are obtained from strains by using the constitutive law of the material and often are far from accurate.

In structural theories such problems are easily overcome, but finite element models require novel strategies. By disregarding specific proposals that are confined to particular elements or situations, we shall mention general approaches only. In contrast to engineering theories, the displacement model of the finite element formulation does not possess any physical meaning and, hence, it loses its privileged character. Other fields, such as stresses, strains or part of them, can be modeled, giving rise to equilibrium, mixed or hybrid formulations. In the displacement based approach, the potential energy theorem rather than the principle of virtual work was employed to formulate the problem and variational statements of energy nature were introduced and used also for alternatives.

The most popular among them are mixed methods, which supplement the displacement model with that of other fields and are based on mixed variational theorems. Some of these statements were established prior to the advent of the finite element method, but little effort was devoted toward their use for structural mechanics computation purposes. A notable exception is the plate solution given by Reissner [8], obtained on the basis of a mixed variational theorem produced to this purpose. It is interesting to observe that the theorem was not new, since a more general version of it was proposed by Hellinger thirty years before [9]. The fact that Reissner ignored this result clearly indicates how limited was the consideration given to these theorems at the time.

Presently, on this statement, usually referred to as the HellingerReissner theorem, are based several mixed finite element models, which proved successful in overcoming some of the drawbacks of displacement approaches, notably in improving the local stress representation. But the search for finite element solutions also produced novel variational statements. Among them one must mention Hermann theorem [10], which can be effectively used to avoid locking phenomena in incompressible or nearly incompressible bodies, a result achieved by decoupling volumetric and isochoric contributions and imposing the 
incompressibility constraint by means of Lagrange multipliers. Since problems with incompressible bodies might arise also in continuum solutions, Herrmann theorem can be regarded as a significant contribution given by finite element methods to structural mechanics as a whole.

The considerations above are a synthetic summary, certainly partial and oversimplified, of the enormous amount of research that took place as soon as the finite element method entered the scene. Early developments were often disordered and sometimes chaotic, but formulations based on sound mechanical concepts were eventually arrived at. As far as the elastic range is concerned, the matter of mathematical calculation mentioned by Love could be considered as solved by about 1970 .

\section{BEYOND THE ELASTIC RANGE}

The success achieved by finite element schemes in solving elastic problems made the engineering community greedy and linear elasticity pretty soon became a too narrow domain. Extensions beyond the elastic range were attempted as soon as the reliability of linear computations was assessed. The existence of satisfactorily performing elastic codes led to inelastic formulations that could fit into them with limited modifications. Hooke's law was replaced by the relevant nonlinear law, enforced in a more or less empirical fashion, and the nonlinearities in the resultant equations were tackled by means of iterative solutions of linear systems. As a whole, it is not an overstatement to say that in most of the inelastic developments of finite element applications very little use was made of the concepts and principles of nonlinear structural mechanics.

Although simple-minded, these formulations proved successful in several instances and success contributed to divert attention from their mechanical foundations. Much attention was dedicated to the computational efficiency of the iterative solution procedures. General methods, such as Newton-Raphson, were replaced by specific strategies, such as initial strain or initial stress methods. The search for solution algorithms capable of tackling very peculiar behaviors, such as snapback, led to the proposal advanced by Riks [11], the method most robust and today most widely employed for nonlinear analyses. All these procedures, however, essentially consider a nonlinear elastic behavior for the material and do not take into account the fact that 
some inelastic laws, typically those produced by the flow theory of plasticity, are inherently nonlinear even at the incremental level, due to the loading-unloading alternative, so that the structural response coincides with nonlinear elastic one only if plastic deformations are monotonously increasing throughout the body.

Confining attention to stress analysis of elastic plastic structures, the theory of Mathematical Programming appears as a natural basis for numerical solution procedures, since it incorporates the inequality constraints that cannot be eliminated from the plastic flow rule. The incremental elastic plastic problem, when discretized by means of finite elements, is spontaneously led to a mathematical programming formulation [12] and it is no wonder that the mathematical properties of constrained optimization problems have a mechanical counterpart in the theory of plasticity. However, in spite of the conceptual interest of these results, mathematical programming theory has not developed, as yet, stress analysis algorithms competitive with traditional iterative schemes and the success of the latter procedures diverted attention from these formulations.

Another aspect deserving some comment is that the replacement of a linear with nonlinear constitutive law for the material behavior makes it difficult, if not impossible, to enforce it exactly within each element. Some approximations are mandatory and their nature ought to be investigated and understood to assess the element behavior. Usually the law is enforced at some points within the element only, typically the integration points employed to numerically compute the element matrices. The use of a large number of such points might be thought of as more accurate, but it turns out that locking phenomena in displacement models are greatly emphasized. Computational experience shows that the use of the points that would be used for reduced integration gives better results, even if element matrices are computed by complete integration. All these artifices are of an essentially empirical nature and a more detailed study of their implications no doubt is desirable.

As final remark note that the procedure above does not even require the existence of an out-and-out constitutive law. Any stressstrain relationship, even a simple collection of experimental data, can be used and, in fact, several problems with ill defined mechanical properties were numerically solved with apparent success. Extensions to large strains, possibly combined with material nonlinearities, and the presence of discontinuities were also dealt with, so that today solutions 
are available for an enormous number of problems previously unsolvable and even hard to formulate in mechanical terms.

\section{EXPECTATIONS AND WORRIES}

The advent of electronic computation has transformed much of theoretical mechanics into a practical tool and an essential basis for a great number of technological developments. The impact of computers was so sudden that a cultural revolution was felt to be at the door. Because of this fact both great expectations and some amount of worrying arose.

As for expectations, it is of interest to quote some passages of the introduction of a structural mechanics book published in 1960: "The challenge here is to develop completely new methods of structural analysis and design which most efficiently use the full computational capacity of these electronic computers. In general, the analytical procedures which have been evolved over the years for efficient manual computation cannot be expected to be equally effective for machine computation. ... At present, it appears that the simplest approach to the organization of structural calculations for machine solution is the matrix formulation of structural theory using the notation and the operations of matrix algebra. Future considerations and developments may in fact demonstrate that this is the approach which the profession seeks for machine computation. Intuitively it seems, however, that this approach is too traditional and that completely new concepts and procedures are needed to utilize the full potential of machine computation" ([5], pp 33-34).

On the other hand, worries are mainly focused on what Oden and Bathe referred to as the Number Crunching Syndrome, that is the "blatant overconfidence, indeed the arrogance, of many working in the field ... that is becoming a disease of epidemic proportions in the computational mechanics community. Acute symptoms are the naive viewpoint that because gargantuan computers are now available, one can code all the complicated equations of physics, grind out some numbers, and thereby describe every physical phenomena of interest to mankind" [13].

Computational mechanics is now sufficiently well settled to permit an assessment on how much expectation was fulfilled and how much concern was justified. The advent of completely new concepts and procedures has not taken place yet and, even if one has to look at future 
developments with an attitude as little prejudiced as possible by present methodologies, it is not foreseen to occur in the immediate future. Certainly, the ease by which matrix methods can be implemented in computer codes has focused on these formulations a research effort much greater than it was in previous time, but if these approaches are considered to be too traditional one must admit that computational mechanics is still contained inside the conceptual framework of classical structural mechanics, from which it received more than what it is presently able to return.

The statement above is not meant at underrating the achievements that computational mechanics has obtained in such a comparatively short period. Undoubtedly, the most impressive of them is the capability of providing successful solutions for otherwise unsolvable problems, and success has brought about the number crunching syndrome. The disease did develop and still exists and produces damage. Reference [13] gives a detailed account of such aspects and on their implication on the developments of computational mechanics.

An example is the growing attitude toward the foundation of continuum mechanics and applied mathematics, the study of which is considered sometimes as a waste of time for people interested in solving so-called real problems. As it was mentioned previously, the increasing complexity of the situations tackled has brought finite element solutions toward problems with poor mechanical foundations, if any. In most conferences on computational mechanics one can listen to presentations showing solutions of problems of incredible complexity, such as a car crashing on an obstacle and undergoing extremely large permanent deformations or an ensemble of stones rolling down a slope and bouncing with each other. These presentations often concentrate on spectacular details, such as the car door opening because of the impact, but little attention is paid to the meaning of the solution.

Actually, the mere fact that a problem cannot be formulated in a mechanically sound fashion does not mean that it must be disregarded. Such problems exist, often are of relevance and no information on them is available except that from numerical solutions. However such solutions, which are expected to be not unique and very sensitive to the assumptions made and to the initial conditions and configuration, should be discussed critically and used to detect the essential aspects of the phenomenon. In the car crushing problem, the fact that the door 
opens because of the impact is of limited relevance, more significant is the amount of energy dissipated and it would be of interest to assess how it depends on the different assumptions for the strain measures (still the object of discussion in finite strain plasticity) and on the particular form of the constitutive relationships. Similarly in a stone avalanche the fact that two particular blocks hit each other and bounce as a consequence is spectacular but immaterial; of greater significance would be to establish how the place at which the avalanche stops depends on the initial configuration.

The Number Crunching Syndrome does not lie in numerically solving difficult problems, rather in the blatant overconfidence on the results. The fact that numerical solutions often are uncritically accepted not only is naïve; it also prevents their use toward a better understanding of problems that classical structural mechanics cannot tackle. A potentially great contribution of computational mechanics to structural knowledge is lost in this way.

\section{THE IMPACT OF COMPUTATIONAL ON STRUCTURAL MECHANICS}

It is now time to go back to the title of this contribution. By adding a question mark one can ask: Structural mechanics: how computers affected it? Previous considerations would suggest the answer: Very little, if at all. In fact the great (and somehow naive) expectations that arose in early times, envisaging a sort of revolution, the advent of a new mechanics of discrete nature replacing the traditional one, did not take place. On the other hand, the possibility of gaining some knowledge on the mechanical behavior of structures for which only numerical solutions are available was not exploited so far, maybe because of the detrimental effect of the number crunching syndrome. Computational mechanics certainly produced a huge amount of results, but mainly focused on a better exploitation of computer facilities for the solution of problems. These results are important enough for computational mechanics to be considered as an independent field. However the theoretical background of structural mechanics kept essentially unchanged.

Nevertheless some beneficial effects were produced. They did not cause dramatic changes, but introduced some improvements and contributed to clarify a few aspects. One of them is associated to energy 
theorems and energy like mixed variational principles. Most of them were established before the computer age, but the overall picture was somehow confused. Results often were considered individually, rather than inserted within a comprehensive framework. As an example, Castigliano's theorem often was presented as an independent result, rather than an important and non trivial consequence of the complementary energy principle. Mixed variational statements were considered mainly as a theoretical curiosity and largely ignored (Reissner independently rediscovering Hellinger illustrates the situation). Numerical analyses strongly contributed to a better understanding of the problem. Initially progress was rather chaotic, with several "principles" introduced more or less randomly. Some of them were not new, others trivial consequences of existing results. However the situation improved gradually and eventually a comprehensive framework emerged. Within this framework new results, such as Hermmann [10] and Hu-Washizu [14] theorems, found proper place. Today the picture is satisfactorily settled and computational mechanics deserves recognition as the main contributor to this result.

Significantly affected by computational mechanics also were the engineering theories of beams, plates and shells. It was stated previously that these theories, being based on displacement assumptions, drew inspiration for the first developments of the finite element method. This is certainly true in general terms, but the procedure followed by finite element formulations was only adopted by Kirchhoff when establishing his theory of thin plates [3]. Theories of beams (both neglecting and considering shear deformations), plates other than thin, thin walled cross sections and shells were established before the computer age, but were developed independently of each other, without any attempt at establishing mutual relations and at providing a unified framework. As it is recognized today, they all can be based on suitable displacement assumptions and formulated by following the same steps as in finite element approaches (except that for stress recovery). This procedure does not introduce any change in the theories, but it clarifies some aspects and permit extensions beyond the elastic domain, when inelastic solutions are sought.

These are only two examples, by no means exhaustive. However they seem sufficient to show that, if the advent of computers did not affect the essential nature of structural mechanics, a better understanding was gained, which produced non minor benefits. 


\section{REFERENCES}

1. Love A.E.H., A Treatise on the Mathematical Theory of Elasticity, $4^{\text {th }}$ ed., Dover Publications, New York, N.Y., 1944.

2. Benvenuto E., La Scienza delle Costruzioni e il suo Sviluppo Storico, Sansoni, Firenze, Italy, 1981.

3. Kirchhoff G., Uber das Gleichgewicht und die Bewegung einer elastichen Scheibe, J. Reine Angew. Math., 45, p. 51, 1850.

4. Turner M.J., Clough R.W., Martin H.C., Topp I.J., Stiffness and deflection analysis of complex structures, J. Aero. Sci., 23, p. 805, 1956.

5. Norris C.H., Wilbur J.B., Elementary Structural Analysis, $2^{\text {nd }}$ ed., McGraw-Hill, New York, NY, 1960.

6. Hrennikoff A., Solution of problems of elasticity by the framework method, $J$. Appl. Mech., 8, p. A169, 1941.

7. Przemieniecki J.S., Theory of Matrix Structural Analysis, McGraw-Hill, New York, NY, 1968.

8. Reissner E., The effect of transverse shear deformation on the bending of elastic plates, J. Appl. Mech., 12, p.69, 1945.

9. Hellinger E. Die allgemeine Ansatze der Mechanik der Kontinua, in: Encyklopadie der Mathematischen Wissenschaften mit Einschluss ibrer Anwendungen, volume 4, 1914.

10. Herrmann L.R., Elasticity equations for incompressible and nearly incompressible materials by a variational theorem, AAIA Journal, 3, p.1896, 1965.

11. Riks E., An incremental approach to the solution of buckling and snapping problems', Int. J. Solids Struct., 15, p. 524, 1979.

12. Cohn M.Z., Maier G. (edts), Engineering Plasticity by Mathematical Programming, Pergamon Press. New York, N.Y,. 1979.

13. Oden J.T., Bathe K.J., A commentary on computational mechanics, Applied Mechanics Reviews, 31, p.1053, 1978.

14. Washizu K., Variational Methods in Elasticity and Plasticity, Pergamon Press, Oxford, U.K., 1975. 